\title{
Face recognition of Pose and Illumination changes using Extended ASM and Robust sparse coding
}

\author{
Arulmurugan $\mathrm{R}^{1}$, Laxmi Priya M.R ${ }^{2}$

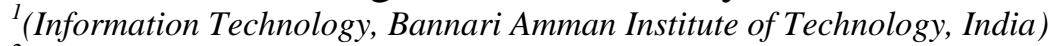 \\ ${ }^{2}$ (Information Technology, Bannari Amman Institute of Technology, India)
}

\begin{abstract}
Face recognition system is an application that is used to identifying or verifying a person from a digital image, which can be done by comparing selected facial features from the image and a facial database, but robust commercial applications are still lacking. Face images used present variations in pose, illumination, image quality, and resolution. The benefits of using image quality and reliability is to improve the accuracy. Face recognition (principal component analysis (PCA) to assess the feasibility of real world face recognition, but the system performance are low when the image in uncontrolled poses. Active shape models (ASMs) are statistical model, which iteratively deform to fit to a new image. The shapes are constrained by the PDM (Point Distribution Model) which is statistical shape model, to vary only in training set of labelled examples. Then weighted matching will be applied between the input image and database images. This method provide the better recognition performance when compare to the Existing methods. Here we detect the face by the Active shape model Algorithm. It is reliable to uncontrolled pose images.
\end{abstract}

Keywords: Pose and Illumination changes, Reliability indices, Image Preprocessing, Sparse coding.

\section{INTRODUCTION}

The face recognition in unconstrained scenarios is still a major challenge. Face recognition has been an active research area and many types of algorithms and techniques have been proposed. Some of the most popular and well-established algorithms for face recognition are principal component analysis (PCA), LDA, ICA, and SVM to assess the feasibility of real world face recognition in uncontrolled setting. In typical surveillance scenarios, people are usually walking free, and they are impossible to always keep their faces frontal or looking to the cameras. Face images captured by surveillance systems are non-ideal, because they are often affected by many factors: pose illumination, expression, occlusion, distance, weather and so on. 3D methods are always based on a 3D face model, which may be a single model, or a deformable model in certain parametric forms. In typical face recognition applications, the enrolled face images (gallery) are usually captured under controlled environment. [1] 3D methods can be divided into four categories depending on how to use of the 3D model:

1. Pose Normalization: Face images in the probe are normalized to frontal view based on the 3D model, and then match the normalized probe to the gallery [8].

2. Pose Synthesis: Use the 3D model to generate some virtual face images with various poses for the face images in the gallery, and then match the probe to the virtual face images.

3. Recognition by Fitting: Fit all face images in the gallery and probe by the 3D model. The texture and shape parameters are used for face recognition [9].

4. Filter Transformation: Transform the filters according to the pose and shape of face image, and then use the pose adapted filters for feature extraction.

When the same face will appears differently due to the variation in lighting and poses changes, the illumination problem will occur and there exists head rotation. The uneven lightning brings variations in illumination which affects the classification greatly since the facial features that are being used for classification gets effected due to this variation.

The Local Binary Pattern (LBP) is an invariant feature extraction type [5] algorithm. It was first proposed for the use of texture description and it has been used to normalize illumination in face detection and recognition contexts. In LBP the center pixel cannot be compared with itself. So LBP cannot capture the local structure of the image. These images should be reduced in the size, to perform this operation, the convex cone. This convex cone is also known as illumination cone. These images are formed from the set of images with the same posture.

This illumination cone can be well approximated by a low-dimensional linear subspace. Under variable lighting, the set of various images are characterized by a illumination cones parameterized by the pose 


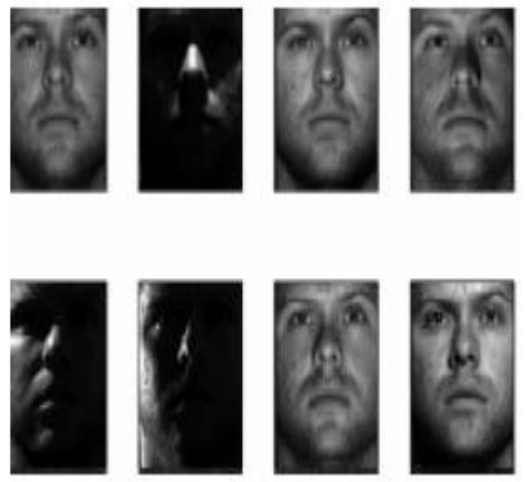

Fig.1. The illumination Problem

\section{LITERATURE SURVEY}

One of the approaches of face description is the texture descriptor where it is used to build several local descriptors of the face and after that it combining them into a global description. In order to motivate for a holistic description [3], the facial image is divided into local regions and texture descriptors which have been extracted from each region independently. The information about the patterns on a pixel-level is carried out by the Local Binary Pattern, the labels are grouped over a small region to produce information of a regional level and then finally regional histograms are concatenated to build a global description of the face.

A variety of algorithms can be tested using the set of new images for calculating performance based on the potential application of face book. The comparison results from PCA, LDA, ICA, and SVMs shows that no single or hybrid method tried is ideally matched to a common application which are used by millions of Face book [4] users. The approach of SVM and LDA methods shows the result of $65 \%$ accuracy with the high cost computation and memory requirements. Later all the tested images are retrained with new image to bring out the original image. Similarly, the Individual PCA approach is used to match the image with a real world implementation but its leads low accuracy [4]. Thus the individual PCA brings out the full sufficiency and also it automatically detect the faces from the database and finally it recognize the original centered face.

\section{IMAGE PREPROCESSING}

\section{Noise Reduction}

A noise reduction filter is used in the binary image, which is used for eliminating single black pixel on white background. Eight neighbors of chosen pixels are examined if the number of black pixels are greater than white pixels then it is considered as black or else white. If there is any dirt on camera, scanner lens, imperfection in the scanner lighting etc, this shows the noise in the scanned face image.

The noise in the image can be removed with the help of filtering function, and works like a common function that replaces each pixel by its function.

\section{Image Segmentation}

The edges from shading have a large effect on the quality of an initial restored image. The edges may come from facial features include eyes, eyebrows, nose and mouth. Therefore, it is necessary to segment these facial feature regions and shading regions in order to reserve only the edges from shading for further processing. As the reconstructed images using PCA have a smaller number of noise points, these images, rather than the initial restored images [2], are used in segmentation for extracting edges. First, we can segment a reconstructed image to obtain the regions of the eyes, eyebrows, nose and mouth using an optimal threshold segmentation method. The presence of noise points should be removed. The uncovered small regions must also to be merged.

To perform these to operations, the erosion and dilation operators are used. In this the raw image is captured under an unknown light source to obtain the dark regions. The dark regions further divided into the darker region by means of the same segmentation method.

\section{Gaussian Filter}

The restored image has a truer appearance if the size of the Gaussian filter is larger. However, the edges from shading and the noise in shading will also be magnified more and will appear in the restored image when a large filter size is used. Therefore, the uneven illumination variation is related to the gray-level intensities in shading and the edge strength from the shading in a raw image. These two factors are therefore 
used as the criteria in the determination of the maximum filter size in order to reduce the uneven illumination variation.

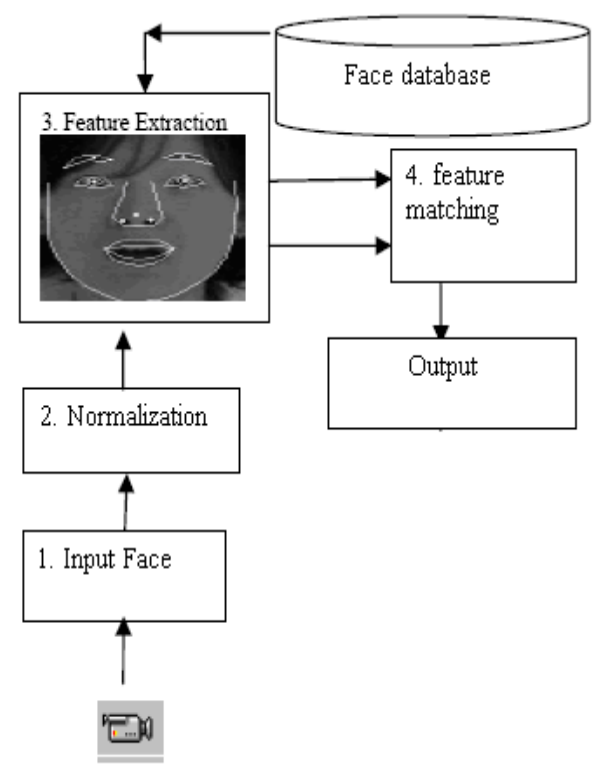

Fig.2. Basic face recognition system

\section{PROPOSED WORK}

Specification Histogram Normalization is one of the most commonly used methods for preprocessing. In image processing, the idea of equalizing a histogram is used to stretch and redistribute the original histogram using the entire range of discrete levels of an image, in a way that an enhancement of an image contrast is achieved.

The image histogram has to be converted to the histogram which is constant for all the brightness values, the technique used here is histogram equalization. This is the most commonly used method in the histogram normalization technique. This would match to a brightness distribution where all values are equally probable. For image $(\mathrm{x}, \mathrm{y})$ with discrete $\mathrm{k}$ gray values histogram is defined by i.e. the probability of occurrence of the gray level $i$ is given by:

$$
p(i)=\frac{n_{i}}{N}
$$

$\mathrm{i} \in 0,1 \ldots \mathrm{k}-1$, level of grayscale image and $\mathrm{N}$, the images pixels (total value).

Transformation to a new intensity value is defined by:

$$
I_{\text {out }}=\sum_{i=0}^{k-1} \frac{n_{i}}{N}=\sum_{i=0}^{k-1} p(i)
$$

Output values are from domain of $[0,1]$.To obtain pixel values in to original domain, it must be rescaled by the $\mathrm{K}^{-1}$ value. The widespread histogram equalization cannot correctly improve all parts of the image. When the original image is irregularly illuminated, some details on resulting image will remain too bright or too dark. These are most commonly used techniques of histogram adjustment. HE is to create an image with uniform distribution over the whole brightness scale and HS is to make the histogram of the input image have a predefined shape.

\section{Active Shape Model Algorithm}

Active Shape Models (ASMs) are automatically locating landmark points that define the shape of statistically modeled object in an image, in order to find the candidate shape and global model to constrain the searched shape the local appearance shape models are used. When modeling faces, the landmark points of interest consist of points that lie along the shape boundaries of facial features such as the eyes, lips, nose, mouth and eyebrows. 
The training stage of an ASM involves the building of a statistical facial model from a training set containing images with manually annotated landmarks. Statistical models of the grey level intensities of the region around each landmark are built using 2D profiles which are generated by sampling the image in a square region around each landmark. Face relighting method based on ratio-images related to different lighting directions by using a set of bootstrap images and image warping by using the active shape model (ASM). This method achieved similar results to the nine-point light source method, but a set of bootstrap images is needed and some feature points must be located accurately.

The above methods require the use of multiple images with different illumination variations as training images, or a set of bootstrap images. In contrast, our algorithm requires only a set of training face images with frontal illumination, and does not need to consider the categories of illumination variation in practical applications.

\section{Feature Extraction}

Feature extraction is concept of extracting the needed data from an image. There are two features available in the feature extraction concept are Global and Grid features. The combination of Global features and Grid features are used to extract the features. The inter ocular distance, the distance between lips and the tip of the nose, the distance between nose tip and the line joining pair of eyes, the distance between lips to the line joining the pair eyes, the eccentricity of the face, ratio of dimension, width of lips all these fall under the Global features. Some of the features of the face images will have the skin color, moustache region, lip region, eye tail, fore head, canthus, eyelid, and nose wing. These features of the face image were considered under the grid features. In this, face can be detected by using Active shape model Algorithm.

\section{FACE MATCHING}

The sample pose and sample illumination for pose and illumination respectively, and normalization process that corrects estimated pose and illumination changes, and then the second module of FACE, dealing with identification and decision making. The matching algorithm and the reliability are used for the matching scores.

\section{Matching Algorithm}

The pose and illumination changes affect different parts of the face in different fashions. The changes experienced are thus local in nature and require individualized processing. In its global form, given two images $A$ and $B$ and the respective mean values of their pixels, $A$ and $B$, respectively, their correlation s(A, B) is defined as

$$
s(A, B)=\frac{\sum_{i=0}^{n-1} \sum_{j=0}^{m-1}(A(i, j)-\bar{A})(B(i, j)-\bar{B})}{\sqrt{\sum_{i=0}^{n-1} \sum_{j=0}^{m-1}(A(i, j)-\bar{A})^{2} \sum_{i=0}^{n-1} \sum_{j=0}^{m-1}(B(i, j)-\bar{B})^{2}}}
$$

The global correlation $S(A, B)$ is obtained as the sum of those local maxima. The number of sub regions in $\mathrm{B}$ that are analyzed for each sub region in A affects both matching precision and its computational cost. A constant value $d$ is chosen such that, given the region in A which starts at position $(x, y)$, it is matched against all regions in B starting at positions $(x+i, y+j)$, with $i=-d$. . d and $j=-d, \ldots, d$. For the decision making the results obtained must be reliable. To result's reliability is assessed during the presence of ranking and matching scores. This is fed to the decision-making stage in order to filter out unreliable authentications and to recognize only those sufficiently reliable.

\section{Experimental Result}

The different sets of experiments are used to assessing different aspects of face. The set of different database of face images were used as a test bed as well as the set of techniques were compared. 


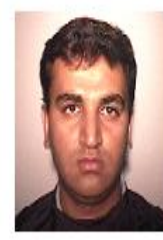

(a)

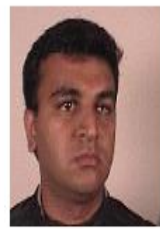

(e)

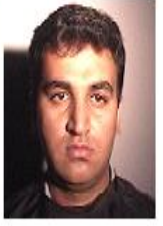

(b)

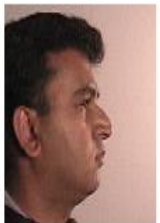

(f)

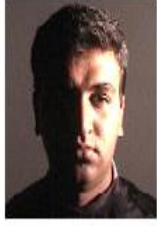

(c)

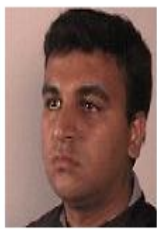

(g)

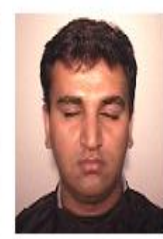

(d)

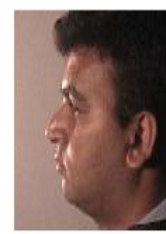

(h)

Fig.3. Dataset of Face pix: a) Ordinary Image b) Shadow Image c) Illumination d) Closed Eyes e), f), g), h) Variations in the pose

Initially we have collected 450 images from the Face pix data set. Using these images we have applied all the preprocessing steps, which are necessary for our project. Then the feature extraction is performed using Active Shape Model (ASM) in which the features are extracted and it is given as a input to the neural classifier. Here, Support Vector Machine has been used for the purpose of classification.

The following table describes about the accuracy of the various methods

TABLE I: Accuracy of Methods across three Datasets

\begin{tabular}{|l|l|l|l|l|}
\hline \multirow{2}{*}{ DB } & \multicolumn{4}{|c|}{ Method } \\
\cline { 2 - 5 } & FACE & ICA & PCA & ASM \\
\hline FACE PIX & $68 \%$ & $79 \%$ & $80 \%$ & $87 \%$ \\
\hline FERET & $87 \%$ & $55 \%$ & $29 \%$ & $42 \%$ \\
\hline LFW & $61 \%$ & $45 \%$ & $48 \%$ & $50 \%$ \\
\hline
\end{tabular}

Even though, there are various methods available to perform the feature extraction, ASM gives the better accuracy compared to all other methods.

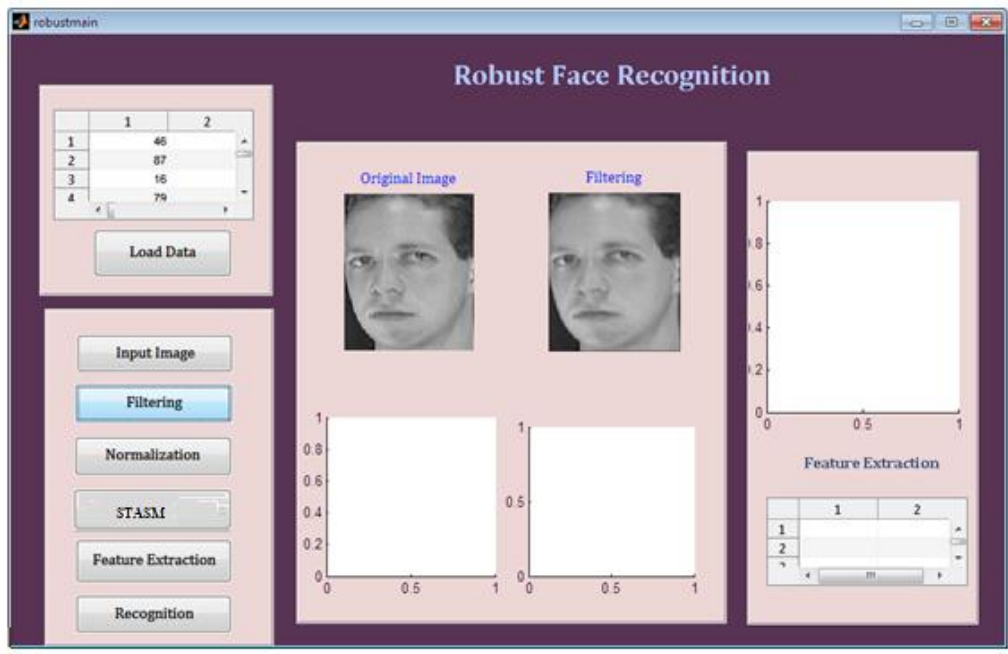

Fig.4. Execution of preprocessing step 


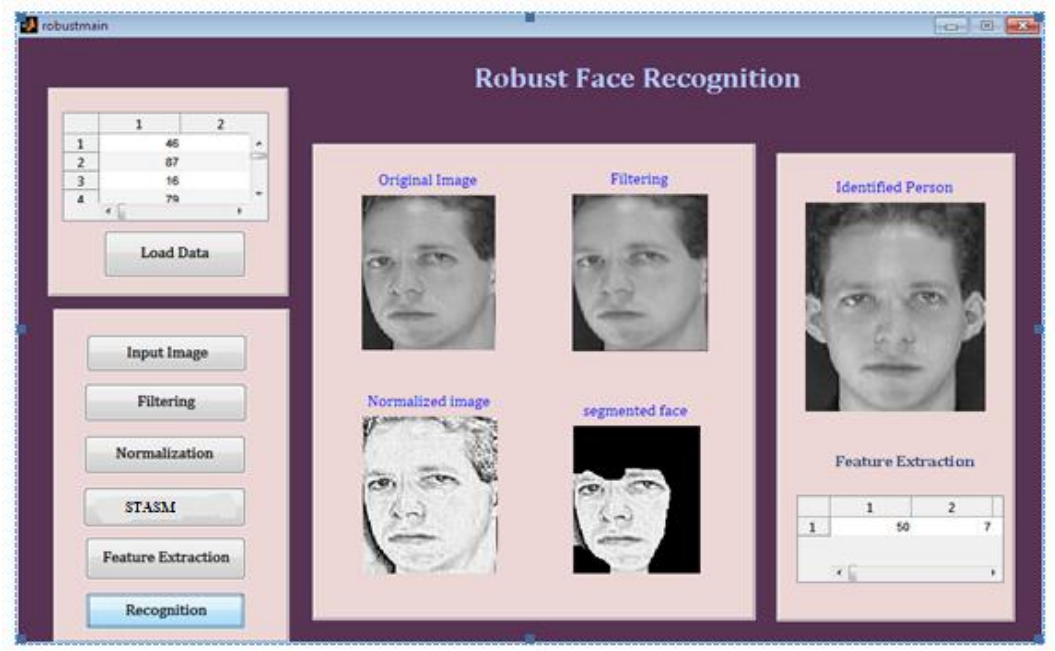

Fig.5. Execution of face recognition step

This figure shows the performance which is obtained by robust face recognition method and gives the better result.

\section{Conclusion And Future Work}

Our method provide the better recognition performance when compare to the existing methods. Here we detect the face by the Active shape model Algorithm. It is reliable to uncontrolled pose images. For classification, the SVM is used and this is more reliable result is obtained. In future the Sparse coding algorithm will be used for feature extraction instead of Active Shape Model.

\section{ACKNOWLEDGEMENTS}

The author is grateful to the anonymous reviewers for their constructive comments, which helped to improve this paper

\section{REFERENCES}

[1] Maria De Marsico, Member, IEEE, Michele Nappi, Daniel Riccio, and Harry Wechsler, Fellow, IEEE

[2] N. Otsu, A threshold selection method from gray-level histograms, IEEE Trans. Systems, Man, and Cybernet. 9 (1) (1979) 62-66.

[3] A. F. Andera, and N. Michele, 2007, "2D and 3D face recognition: A survey," Pattern Recognition Letters, Vol. 28, pp.1885-1906.

[4] J. C. Gower, "Generalized Procrustes Analysis," Psychometrika, vol. 40, no. 1, pp. 33-51, March 1975.

[5] E. Oja, 1983, "Subspace Methods of Pattern Recognition," Research studies Press ltd.

[6] N. Dalal and B. Triggs, "Histograms of oriented gradients for human detection," in Proc. CVPR, Washington, DC, 2005, pp. 886893.

[7] C. Nakajima, M. Pontil and T. Poggio, "People Recognition and Pose Estimation in Image Sequences", Proceedings of the IEEEINNS-ENNS International Joint Conference on Neural Networks, vol. 4, pp. 4189- 4195, July 2000.

[8] A. Asthana, T. K. Marks, M. J. Jones, K. H. Tieu, and M. V. Rohith. "Fully automatic pose-invariant face recognition via 3d pose normalization". In Proceedings of IEEE International Conference on Computer Vision, pages 937 944, 2011.

[9] V. Blanz and T. Vetter. "Face recognition based on fitting a 3d morphable model". IEEE Transactions on Pattern Analysis and Machine Intelligence, 25(9):1063-1074, 2003.

[10] S.Anila \& Dr.N.Devarajan, "An efficient Preprocessing Technique under difficult Lighting Conditions," in Proc. National Conference on Emerging Trends in Computer Communication and Informatics (ETCCI-2011), March 10-11, 2011.

[11] V. Blanz and T. Vetter. Face recognition based on fitting a 3D morphable model. IEEE Transactions on Pattern Analysis and Machine Intelligence, 25(9):1063-1074, 2003

[12] W.Y. Zhao, and R. Chellappa, 2000, "Illumination insensitive face recognition using symmetric shape-from shading," IEEE Conference on Computer Vision and Pattern Recognition, vol.1, pp. 286-293. 